\title{
Understanding SDBD Actuators: An Experimental Study on Plasma Characteristics
}

\author{
R. Geuns*, S. Goekce ${ }^{\dagger}$, G. Plyushchev ${ }^{\ddagger}$ and P. Leyland ${ }^{\S}$ \\ Ecole Polytechnique Fédérale de Lausanne (EPFL), CH-1015 Lausanne, Switzerland \\ R. Pimentel ${ }^{\mathbb{1}}$ \\ A. deChamplainll and Y. Jean** \\ DRDC, Valcartier, Québec, G3J 1X5, Canada \\ Université Laval (UL), Québec, G1V 0A6, Canada
}

\begin{abstract}
The working mechanisms of surface dielectric barrier discharge (SDBD) plasma actuators foreseen as aerodynamic control devices is investigated experimentally on a common platform, referred to as the NATO-AVT-RTO-190 test case. A better understanding of the working principle and characteristics of SDBD paves the way for more efficient and safe use of plasma actuators in aerodynamic applications. In this study, a characterisation of the plasma is done by current measurements, fast-camera plasma imaging and force measurements. Furthermore, more advanced plasma characteristics such as reduced electric field and excited species population are found by Optical Emission Spectroscopy. The collective goal of this research is to contribute to a database which can also be used for numerical verification and validation by varying the key parameters such as frequency and voltage.
\end{abstract}

\section{Nomenclature}

$n_{i} \quad$ Excited species population density, $\mathrm{cm}^{-3}$

$n_{e} \quad$ Electron density, $\mathrm{cm}^{-3}$

$X_{i} \quad$ Target gas density, $\mathrm{cm}^{-3}$

$K_{i} \quad$ Production rate, $\mathrm{cm}^{3} \cdot \mathrm{s}^{-1}$

$E / N$ Reduced electric field, $\operatorname{Td}\left(10^{-17} \mathrm{~V} \cdot \mathrm{cm}^{2}\right)$

$\tau_{0}^{i} \quad$ Radiative lifetime of excited state $i, \mathrm{~s}$

$\tau_{i} \quad$ Total lifetime of excited state $i, \mathrm{~s}$

$Q_{X}^{i} \quad$ Quenching rate of excited state $i, \mathrm{~cm}^{3} \cdot \mathrm{s}^{-1}$

$\varepsilon \quad$ Kinetic energy of electron, $\mathrm{eV}$

$F_{0} \quad$ Electron energy distribution function, $\mathrm{eV}^{-3 / 2}$

$\sigma_{i}^{e} \quad$ Excitation cross section, $\mathrm{cm}^{2}$

\section{Introduction}

Active aerodynamic control using surface dielectric barrier discharge actuators is a promising concept which has been extensively studied over the last decade. Their advantages include fast response, thus enabling real-time control at high frequencies, and simplicity due to the lack of moving mechanical parts.

\footnotetext{
*M.Sc. Student, Interdisciplinary Aerodynamics Group, AIAA Student Member.

${ }^{\dagger}$ Ph.D. Student, Group of Thermal Turbomachinery.

${ }^{\ddagger}$ Scientist, Interdisciplinary Aerodynamics Group.

$\S$ Group Leader, Interdisciplinary Aerodynamics Group, AIAA Member.

`Defence Scientist, Advanced Weapons Concepts Group, DRDC Valcartier.

"Professor, Université Laval.

${ }^{* *}$ Scientist, Université Laval.
} 
At low flow speeds, DBD actuators driven by AC waveforms (AC-DBD) have shown to be efficient due to the production of an ionic wind close to the wall, adding momentum to the boundary layer. Recent reviews by Corke et al. ${ }^{1}$ and Moreau et al. ${ }^{2}$ describe these effects in detail. At higher flow velocities, this equivalent wall-jet does not seem to produce significant effects. In a previous study at EPFL, Pavon et al. ${ }^{3,4}$ investigated the interaction between an AC-DBD actuator mounted on a NACA 3506 profile in transonic flow, where no noticeable effect of the actuator on the shock wave buffeting and strength was observed. However, a strong effect on the discharge plasma state was produced with increasing speeds.

Alternatively, studies have been performed on nanosecond pulsed actuators (ns-DBD), showing effects for transonic flows at very high voltage levels. ${ }^{5,6}$ For these actuators, the working mechanism is believed to be the generation of compression waves that emerge from the surface, adding pulsed energy to the flow. Little et al. ${ }^{7}$ and Grech et al. ${ }^{8}$ have investigated the application of ns-DBD actuators to delay separation for lower flow velocities, up to $u_{\infty}=62 \mathrm{~m} / \mathrm{s}$, where the generated compression wave acts as an active trip at pre-stall angles of attack. The plasma produced here is essentially a streamer discharge regime propagating along the surface of the dielectric.

From these considerations, the relevance of understanding the plasma regimes and transitions in such DBD is clear. In order to further develop safe and effective applications of DBD, research on the characterisation of these plasmas needs to be performed. The NATO-AVT-RTO-190 working group has suggested a standard SDBD setup to investigate the basic characteristics of these plasmas and to evaluate the force and relative power produced, with respect to different input voltages and frequencies from $\mathrm{AC}$ or nanosecond pulsed power supplies. The effects of ambient pressure were also investigated at EPFL by placing the DBD in a low-pressure chamber where the pressure levels of the air can be modulated.

In this work, spectroscopy and plasma imaging are performed under different operating conditions (i.e. voltage amplitude, voltage frequency and ambient pressure) at EPFL. Force and power measurements are performed for different voltages and frequencies at atmospheric pressure at UL.

In section II, the NATO-AVT-RTO-190 experimental setup as used by the authors is described. In section III, results of current measurements are presented. Afterwards, force and power measurements are shown for different frequencies and voltages and plasma imaging is performed to investigate the evolution of the plasma state during the AC cycle. The section concludes with a spectral analysis of the spectroscopy results.

\section{Experimental Setup}

A wide variety of possible dielectrics and electrode materials is available for the manufacturing of DBD actuators. The proposed test case considers a DBD setup of copper electrodes on an acrylic type dielectric, PMMA, set up as in figure 1. The thickness of the dielectric plate equals $3 \mathrm{~mm}$ and suitable silicon based glue is used to attach the copper electrodes.

High voltage is supplied to the actuator by a PM04015-H-CE TREK high-voltage function generator (600 VA max) which provides $\pm 10 \mathrm{kV}$ in $\mathrm{AC}$ or DC mode. The TREK power generator is used in amplifier mode, being triggered by a pulse signal from a SCF-AL-018 RohdeESSchwarz function generator. The sine waveform is used for the experiments.

In order to be able to measure the plasma characteristics at different ambient pressures, the DBD actuator is installed in a low-pressure chamber. A vacuum pump is used to set the pressure in the chamber. Three different pressures are used during the

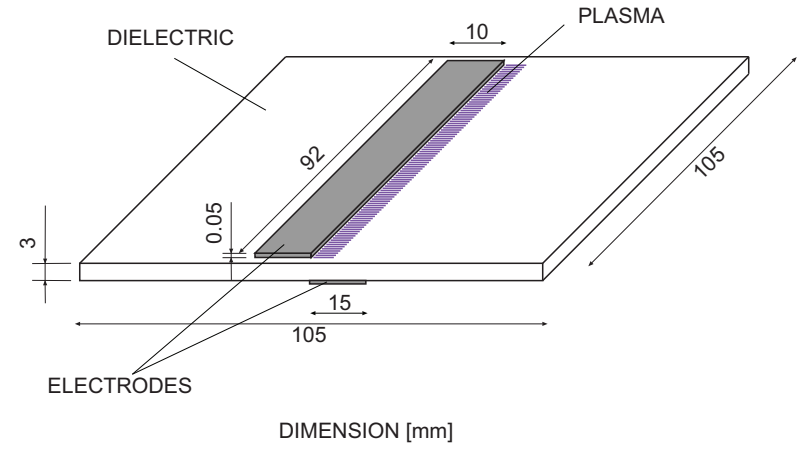

Figure 1. Drawing of the DBD actuator as used in the experiments. Dimensions in $\mathbf{m m}$. 
experiments, namely atmospheric pressure, 500 mbar and 250 mbar. The pressure is measured using a $M K S$ Baratron 626A pressure gauge (1-1000 mbar).

A Princeton Instruments Acton SP2750 spectrometer coupled to an ICCD PI-MAX2 fast camera is used for time-resolved spectroscopy. Light emission originating from the plasma is collected through a converging lens with a focal length of $50 \mathrm{~mm}$ and captured by a row of 16 fibres. The light is diffracted using a grating of $600 \mathrm{~g} / \mathrm{mm}$. At high spectral ranges, a high-pass filter $(>400 \mathrm{~nm})$ is used to filter out the lower wavelengths and avoid second order spectra. The whole optical system is schematically represented in figure 2 . The setup is calibrated in intensity using a Labsphere integrating sphere.

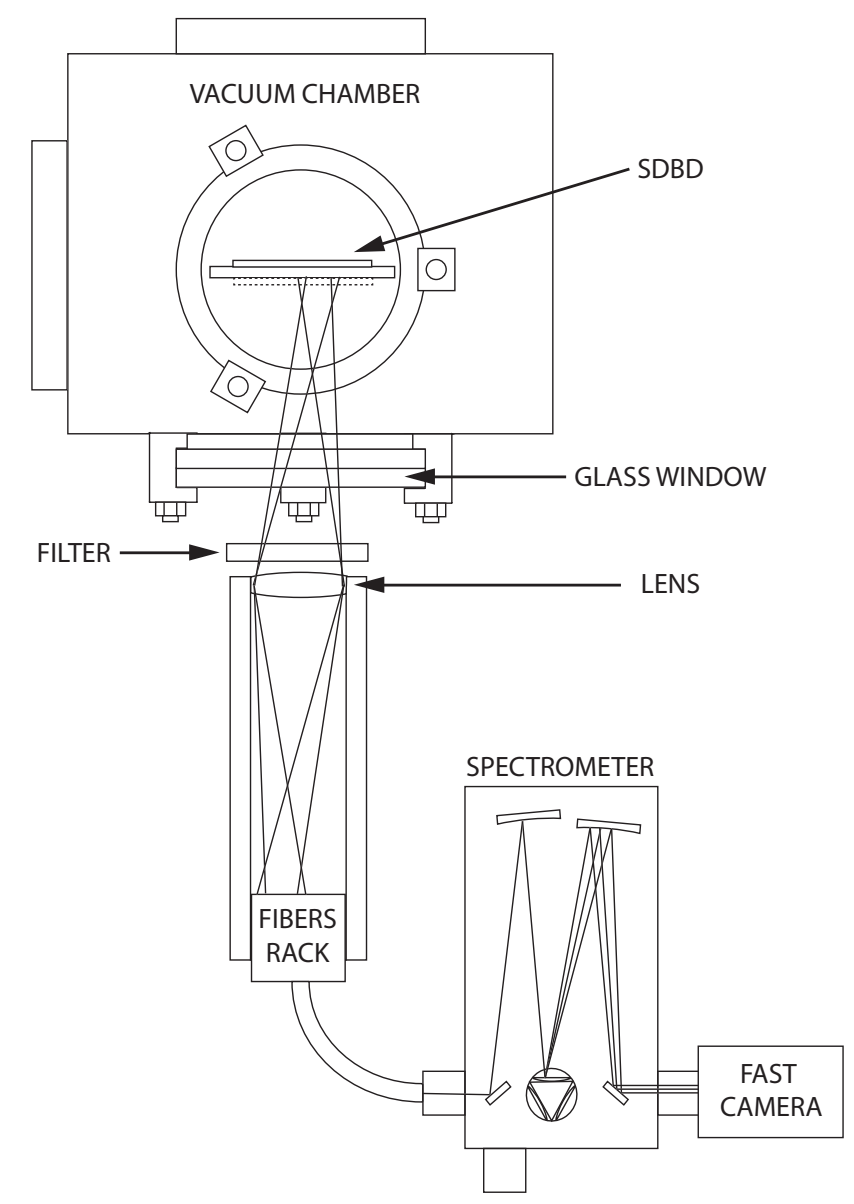

Figure 2. Schematic representation of the spectroscopy optical setup.

A Rogowski coil (Bergoz FCT-016-10:1-WB) is used to measure the current signal over time. The frequency response of this coil equals $700 \mathrm{MHz}$. Data are retrieved from a LeCroy Wavesurfer 454 oscilloscope with a temporal resolution of $4 \mathrm{~ns}$.

Direct force measurements were obtained using a precision balance Ohaus Adventurer Pro AV313C (0.001 g precision). For power supply, a combination of an audio amplifier QSC RX2450 with a Corona Magnetic Inc. transformer was used.

\section{Results and Discussion}

For different actuation frequencies, voltages and operating pressures, the following measurements are performed:

- Current signal measurements to identify power consumption and temporal characteristics of the plasma 
- Force measurements and power efficiency evaluation at atmospheric pressure

- Phase-resolved plasma imaging to visualize streamer propagation

- Time-resolved characterisation using optical emission spectroscopy in order to better understand the state of the plasma.

\section{III.A. Current signal measurements}

Current signal measurements are performed to identify the power consumption by the plasma. Also, these measurements provide useful information about the time span in which streamers are present. In figure 3, a typical DBD current curve is shown. For reference, the measured high voltage signal generated by the amplifier (red line) is plotted together with the reference voltage from the function generator (blue line). While voltage is decreasing, the current disturbances start at about $30 \%$ of the cycle and end at approximately $50 \%$. This is referred to as fall time, whereas rise time indicates an increasing voltage. In case of a symmetric DBD actuator, the positive peaks during rise time are similar to the negative peaks during fall time.

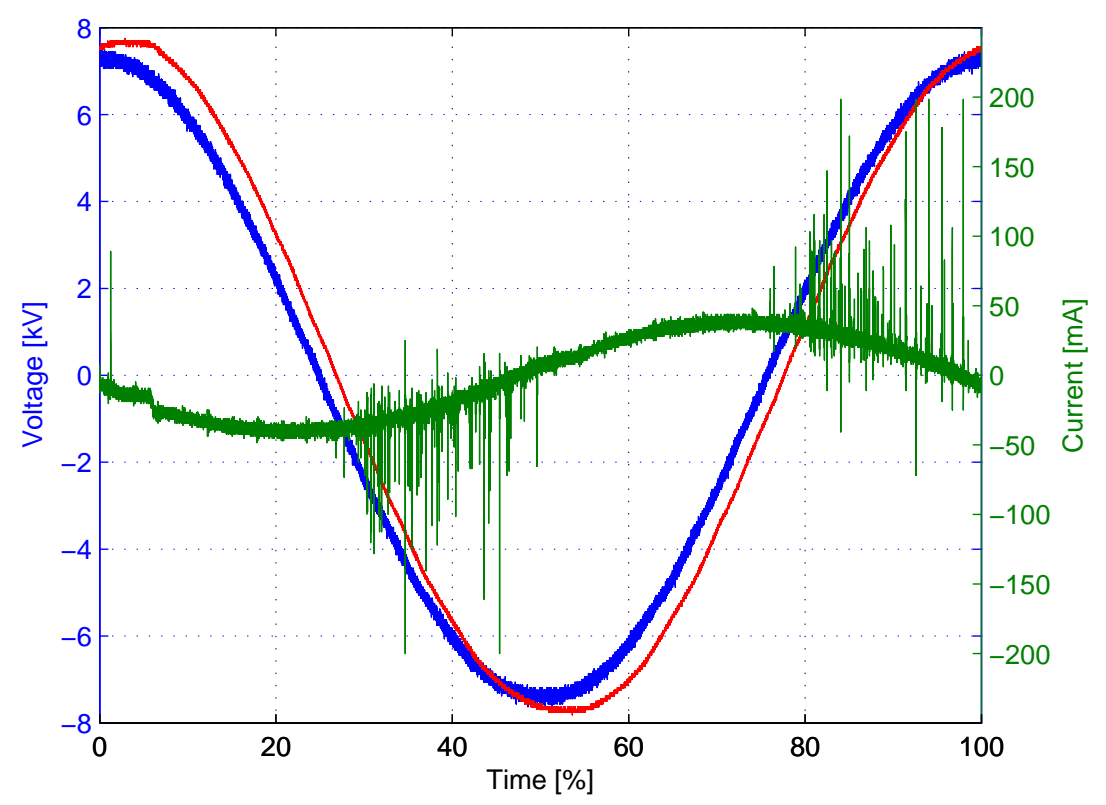

Figure 3. Current (green), measured voltage (red) and reference voltage (blue) versus cycle. Measurements are performed at atmospheric pressure, $15 \mathrm{kV}$ and $2.5 \mathrm{kHz}$.

The current signal encounters ringing, induced by the external electric circuit, which also slightly disturbs the voltage signal. ${ }^{9}$ In figure 3 , this induces small positive peaks during fall time and negative peaks during the rise time of the voltage signal. The capacitance of the DBD actuator changes with varying current, leading to a change in impedance of the electric circuit and disturbance of the voltage signal.

\section{III.A.1. Effect of operating conditions on current signal}

Under different operating conditions investigated, i.e. voltage amplitude, frequency and operating pressure, the current signal differs. Investigating this current signal reveals properties of the plasma. The amplitude and duration of the peaks is of interest, as it gives an indication of the power consumed. As noise is present in the signal measurements, only peaks which are higher than four times the standard deviation of the current signal are taken into account. The integral of these peaks is calculated. Also the duration and starting time of the streamers are derived from the current signal. In table 1, the aforementioned values are presented. 


\begin{tabular}{|l|l|c|c|c|}
\hline & & Unit & $\mathbf{1 0} \mathbf{~ V V}$ & $\mathbf{1 5} \mathbf{~ k V}$ \\
\hline \multirow{3}{*}{$\mathbf{2 . 5} \mathbf{~ k H z}$} & Integrated negative peaks & $\mathrm{mA} \cdot \boldsymbol{\mu} \mathrm{s}$ & 37.4 & 136.9 \\
& Number of negative peaks & & 13.0 & 39.8 \\
& Duration of signal & $\mu \mathrm{s}$ & 77.8 & 101.1 \\
& Start of signal & $\mu \mathrm{s}$ & 127.6 & 109.6 \\
\hline \multirow{3}{*}{$\mathbf{3 . 5} \mathbf{~ k H z}$} & Integrated negative peaks & $\mathrm{mA} \cdot \boldsymbol{\mu s}$ & 51.2 & 182.3 \\
& Number of negative peaks & & 27.9 & 79.0 \\
& Duration of signal & $\mu \mathrm{s}$ & 56.1 & 73.0 \\
& Start of signal & $\mu \mathrm{s}$ & 94.8 & 79.6 \\
\hline
\end{tabular}

Table 1. Statistics of current signal on a symmetric DBD actuator for different operating conditions. Measurements performed at atmospheric pressure.

An increase in voltage amplitude leads to an increase of the mean amplitude of the current peaks. Also, the plasma formation starts earlier with rising voltage amplitude and the power consumption has a longer duration. A possible explanation for this is that the breakdown voltage which is needed to initiate plasma generation has been reached earlier in the voltage cycle. Another observation is that the current peaks end when the maximum voltage is reached.

An increase in voltage frequency also increases the mean value of the peaks. On the other hand, it decreases the duration of the plasma streamers and shifts the starting point towards the beginning of the cycle. This can be related to the new shape of the higher frequency signal. As frequency becomes higher, cycle duration becomes shorter and the voltage slope is steeper. Due to this, the breakdown voltage will be reached earlier.

Another set of experiments was conducted using the same DBD actuator on which one electrode (15 mm width) was covered by dielectric tape. The results of these measurements are listed in table 2 . As one electrode is covered, the DBD actuator is not symmetric any longer. This leads to a current signal which is not similar during rise and fall time of the voltage signal.

\begin{tabular}{|l|l|c|c|c|c|c|}
\hline & & Unit & $\mathbf{1 0} \mathbf{~ k V}$ fall & $\mathbf{1 0 ~} \mathbf{k V}$ rise & $\mathbf{1 5} \mathbf{~ k V}$ fall & $\mathbf{1 5} \mathbf{~ k V}$ rise \\
\hline \multirow{3}{*}{$\mathbf{2 . 5} \mathbf{~ k H z} z$} & Integrated peaks & $\mathrm{mA} \cdot \mu \mathrm{s}$ & -3.5 & 31.1 & -32.1 & 103.5 \\
& Number of peaks & & 5.5 & 17.6 & 32.3 & 53.9 \\
& Duration of signal & $\mu \mathrm{S}$ & 47.0 & 82.4 & 89.0 & 96.5 \\
& Start of signal & $\mu \mathrm{s}$ & 149.5 & 321.7 & 116.9 & 312.0 \\
\hline \multirow{3}{*}{$\mathbf{3 . 5} \mathbf{~ k H z}$} & Integrated peaks & $\mathrm{mA} \cdot \mu \mathrm{s}$ & -1.0 & 52.2 & -36.2 & 180.0 \\
& Number of peaks & & 2.7 & 30.2 & 37.7 & 95.3 \\
& Duration of signal & $\mu \mathrm{s}$ & 32.3 & 70.1 & 62.2 & 72.8 \\
& Start of signal & $\mu \mathrm{s}$ & 103.8 & 231.8 & 88.5 & 220.8 \\
\hline
\end{tabular}

Table 2. Statistics of current signal on an asymmetric DBD actuator for different operating conditions. Measurements performed at atmospheric pressure. Integrated peaks and number of peaks considers positive peaks for the rise time and negative peaks for the fall time.

\section{III.B. Force and power efficiency measurements}

For the thrust and power measurements, the asymmetric DBD actuator with covered electrode is used (uncovered and covered electrodes have widths of 5 and $15 \mathrm{~mm}$ respectively, the length of both electrodes is equal to $155 \mathrm{~mm}$ ). The thrust and power consumption results are normalized by unit length of electrodes. In figure 4 the results of normalized force for different driven voltage and frequency are presented. In this figure it is shown that force generated by the actuator increases as the driven frequency or voltage increases.

The power consumption also increases with driven frequency or voltage, as can be seen in figure 5. From this figure, it can be found that, during the experiments at driven frequencies of $3.5,7.0$ and $14.0 \mathrm{kHz}$, the 


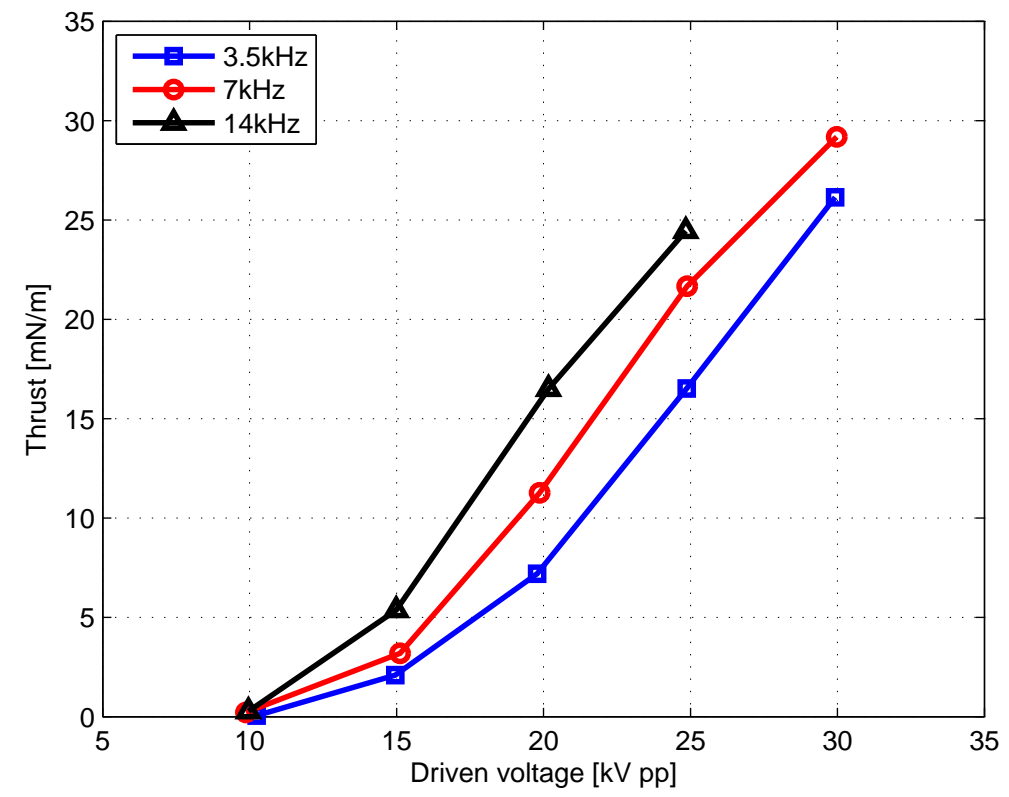

Figure 4. Effect of driven voltage and frequency on the thrust generated by the actuator.

normalized power consumption followed the power-law function with a power exponent varying from 3.4 to 4.0 .

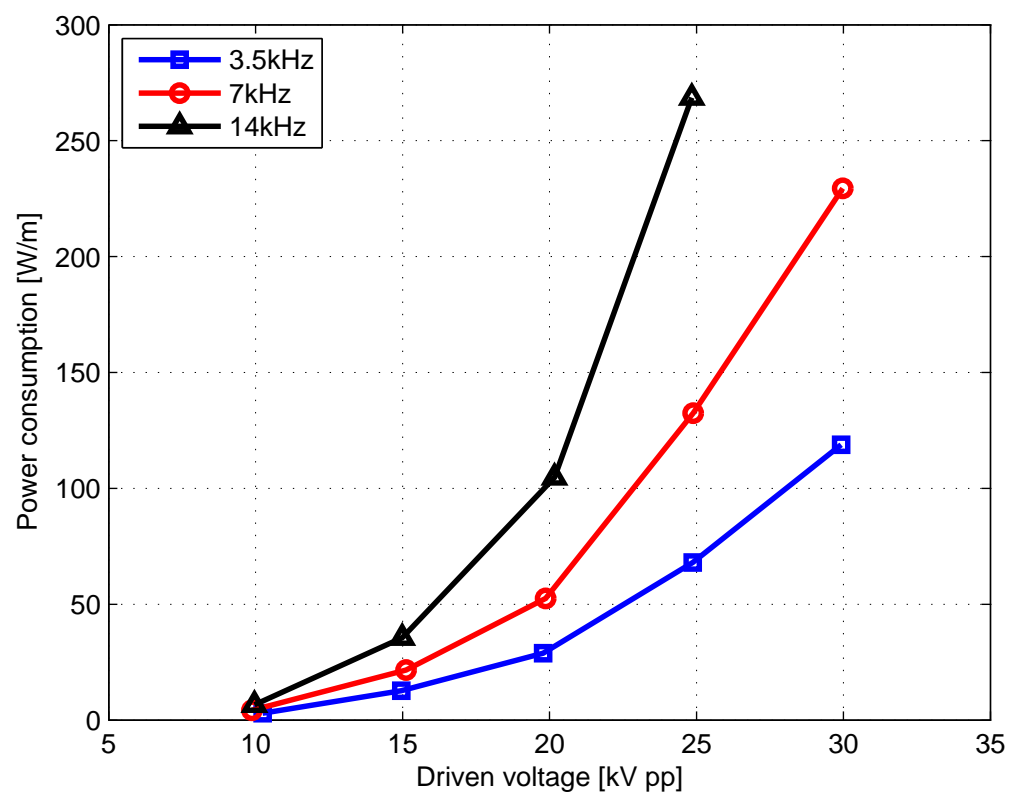

Figure 5. Effect of driven voltage and frequency on the power consumption of actuator.

In figure 6 , the force/power performance parameter is presented. The results suggested that an optimal condition for operating the SDBD plasma actuator exists. For this test case, this optimal is found to be approximately $20 \mathrm{kV}$ peak-to-peak. From this figure, it can be concluded that the lower frequency $(3.5 \mathrm{kHz})$ is more efficient compared to the higher frequencies tested $(7$ and $14 \mathrm{kHz})$ in terms of thrust to power ratio. 


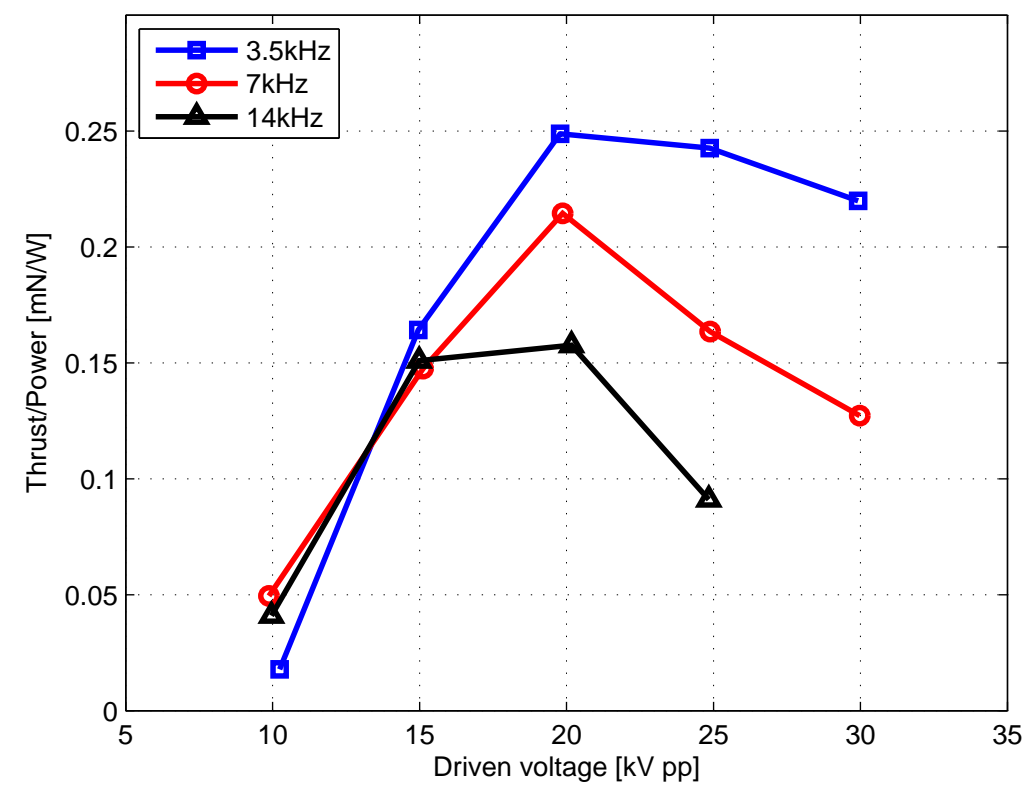

Figure 6. The effect of driven voltage and frequency on the actuator performance.

\section{III.C. Plasma imaging}

In order to better understand the development and propagation of streamers in the air gap between the electrodes, time-resolved synchronized plasma imaging has been performed. Resulting images show a clear distinction between the two phases in figure 3 during rise and fall time of the voltage signal. In figure 7 , examples of images during rise and fall time of the signal are shown. During rise time of the voltage signal (pictures $\mathrm{d}$, e and $\mathrm{f}$ ), the streamers clearly originate at the upper electrode and propagate through the air gap towards the lower electrode. During fall time of the signal (pictures a, b and c), this structure is less clear. Here, the propagation of the streamers is far less pronounced. The plasma extends over a length of about $4 \mathrm{~mm}$ during rise time, whereas it only extends over about $1 \mathrm{~mm}$ during fall time. Nevertheless, both rise and fall time streamers seem to originate at the upper electrode.
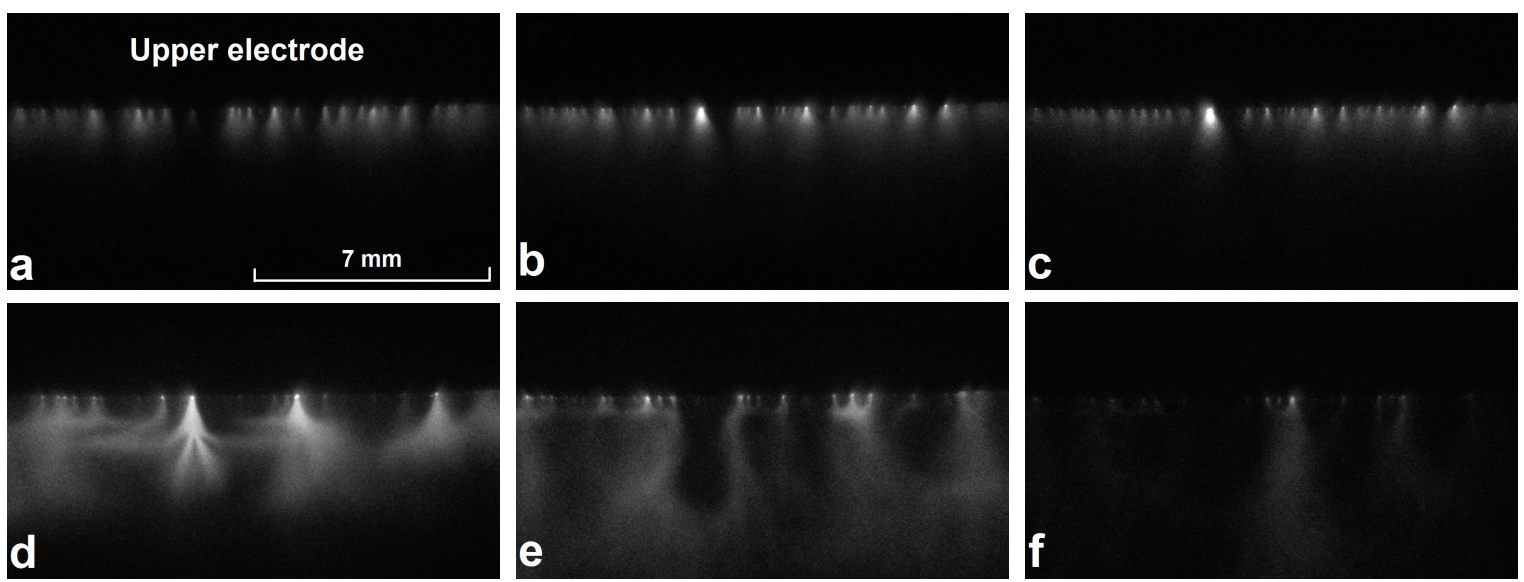

Figure 7. Streamer propagation images during fall (a, b, c) and rise (d, e, f) time of the voltage signal. Pictures a, b and c correspond to 30,40 and $50 \%$ of cycle time respectively. Pictures $d$, e and f correspond to 80,90 and $100 \%$. Voltage amplitude and frequency are $15 \mathrm{kV}$ and $2.5 \mathrm{kHz}$ respectively. Measurements performed at atmospheric pressure.

The difference in streamer mechanisms during rise and fall time of the voltage signal is further elaborated in figure 8 , where the light intensity of the plasma images is plotted against time for two different ambient 
pressures and voltages. A first observation is that the light emission during rise time of the signal is higher than the light emission during fall time in both cases. The peak values for intensity are comparable, which indicates that the maximum light intensity is of the same order for a constant ratio of voltage over pressure. The light emission is initiated earlier at atmospheric pressure and higher voltage compared to the measurements performed at lower voltages. This observation is in agreement with the duration and starting time of the current signal measurements presented in table 1.

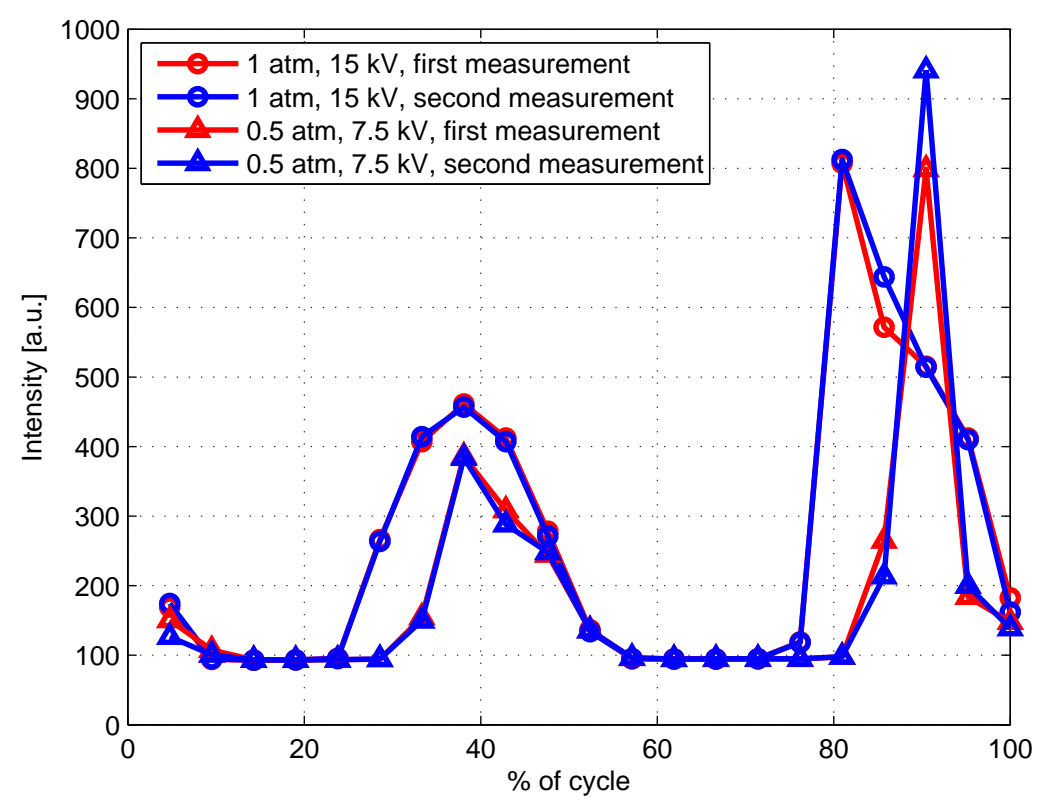

Figure 8. Image intensity over cycle time for voltage amplitudes $15 \mathrm{kV}$ and $7.5 \mathrm{kV}$ at atmospheric pressure and 500 mbar. All measurements performed at $2.5 \mathrm{kHz}$.

Furthermore, in figure 8, two sets of image intensities are plotted using the same conditions for voltage, frequency and ambient pressure. The alignment of the curves is nearly exact, indicating a high repeatability of plasma imaging in terms of intensity.

\section{III.D. Spectral analysis}

Excited species are produced in the plasma, mostly by transfer of energy between energetic electrons and molecules (or atoms). These excited species can de-excite to a state possessing a lower energy, either by colliding with another molecule (quenching) or by radiative decay, i.e. by the release of a photon. The spectrum of this light depends on the electronic structures of the upper and lower states, and therefore allows the unambiguous identification of excited species by analysing the spectrum of the light. The light spectrum emitted by the SDBD was analysed in this study using a spectrometer coupled to a fast camera, as described in the experimental setup in section II.

\section{III.D.1. Spectral features of the plasma}

The most intense part of the spectrum emitted by the plasma is due to the transition $\mathrm{N}_{2}\left(C^{3} \Pi_{\mathrm{u}}-B^{3} \Pi_{\mathrm{g}}\right)$, i.e. the second positive system (SPS) of nitrogen. Besides this transition, also the transition $\mathrm{N}_{2}^{+}\left(B^{2} \Sigma_{\mathrm{u}}^{+}-\right.$ $X^{2} \Sigma_{\mathrm{g}}^{+}$), the first negative system of $\mathrm{N}_{2}^{+}$, was clearly identified. It reveals the presence of the ion $\mathrm{N}_{2}^{+}$. Both transitions can be seen in figure 9 . The spectrum is recorded using light emission collected from 4000 cycles (accumulations). The first positive system of nitrogen (FPS), $\mathrm{N}_{2}\left(\mathrm{~B}^{3} \Pi_{\mathrm{g}}-\mathrm{A}^{3} \Sigma_{\mathrm{u}}^{+}\right.$), and atomic lines due to oxygen and argon were also identified, as shown in figure 10. However, these transitions were much weaker. In order to obtain the same order of intensity as in figure 9, more $(100,000)$ accumulations were needed. 


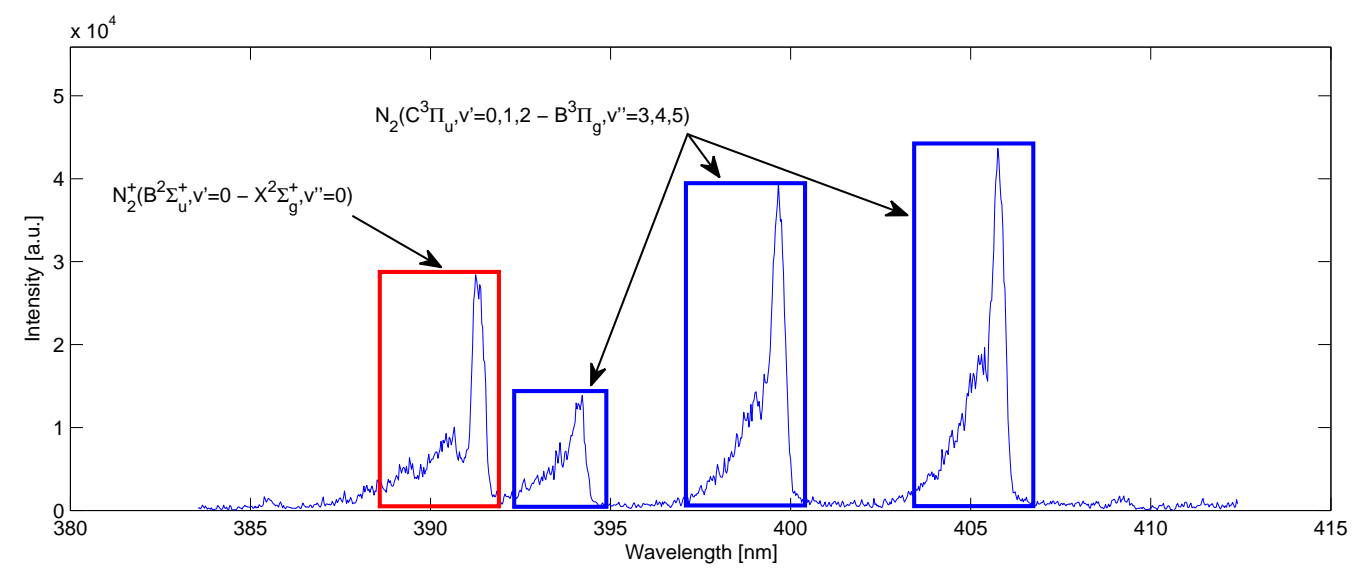

Figure 9. Spectrum at $385 \mathrm{~nm}$ to $410 \mathrm{~nm}$. Voltage and frequency are respectively $15 \mathrm{kV}$ and $2.5 \mathrm{kHz}$. Measurement performed at atmospheric pressure.

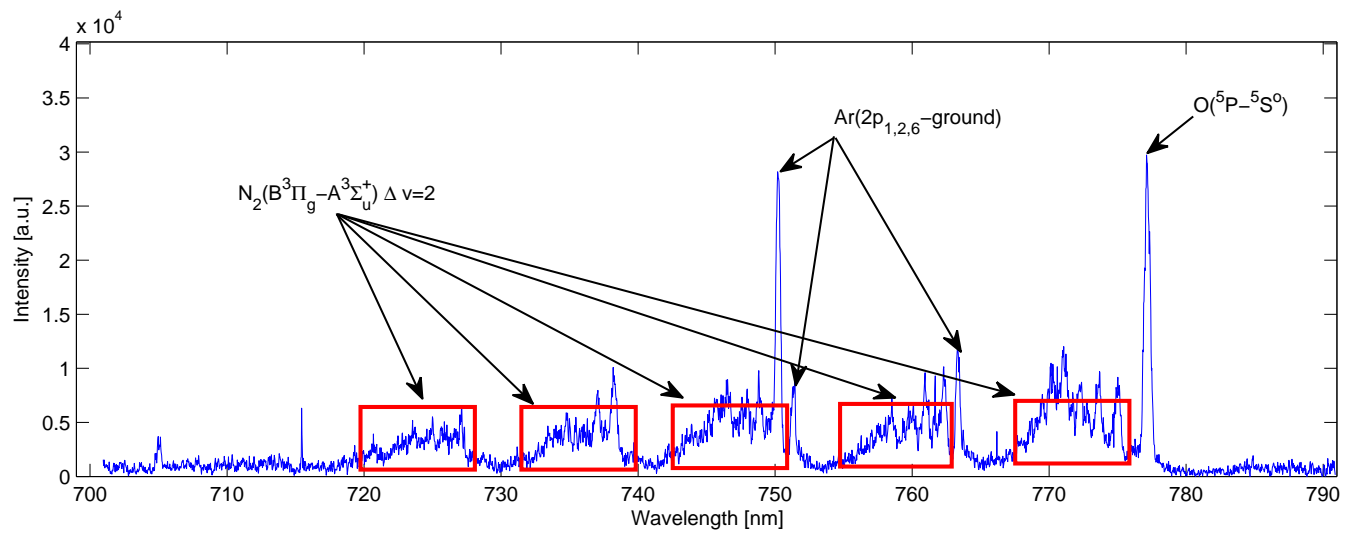

Figure 10. Spectrum at $710 \mathrm{~nm}$ to $790 \mathrm{~nm}$. Voltage and frequency are respectively $15 \mathrm{kV}$ and $2.5 \mathrm{kHz}$. Measurement performed at atmospheric pressure.

\section{III.D.2. Excited species}

The spectra recorded were analysed with an in-house spectral code, able to simulate the spectrum from several spin-allowed transitions of diatomic molecules and some atomic transitions. The determination of the intensities of the rotational lines from the spectra of diatomic molecules are computed following Whiting. ${ }^{10}$ The emission coefficients are taken from Gilmore. ${ }^{11}$ The location of the rotational lines is computed by the method outlined by Hornkohl. ${ }^{12}$ Lambda doubling was neglected apart for the first positive system. The population of excited species was assumed to be at rotational equilibrium but not at vibrational or electronic equilibrium. The vibrational level specific spectra were therefore computed independently. The simulated spectra were fitted to the experimentally recorded spectra using the least square method, which allowed the accurate determination of the population densities of excited species. The resulting excited species populations for the same conditions as figure 9 are shown in figure 11 .

\section{III.D.3. Determination of the reduced electric field}

If the excited species are assumed to be produced only by electron impacts from the ground state and deexcited only by spontaneous radiative decay and two-body collisional de-excitation (quenching), it is possible to determine the average reduced electric field of the plasma. With these assumptions, the evolution of the populations of excited species can be described by Eq. (1).

$$
\frac{\mathrm{d} n_{i}}{\mathrm{~d} t}=n_{\mathrm{e}} X_{i} K_{i}(E / N)-\frac{n_{i}}{\tau_{i}}
$$




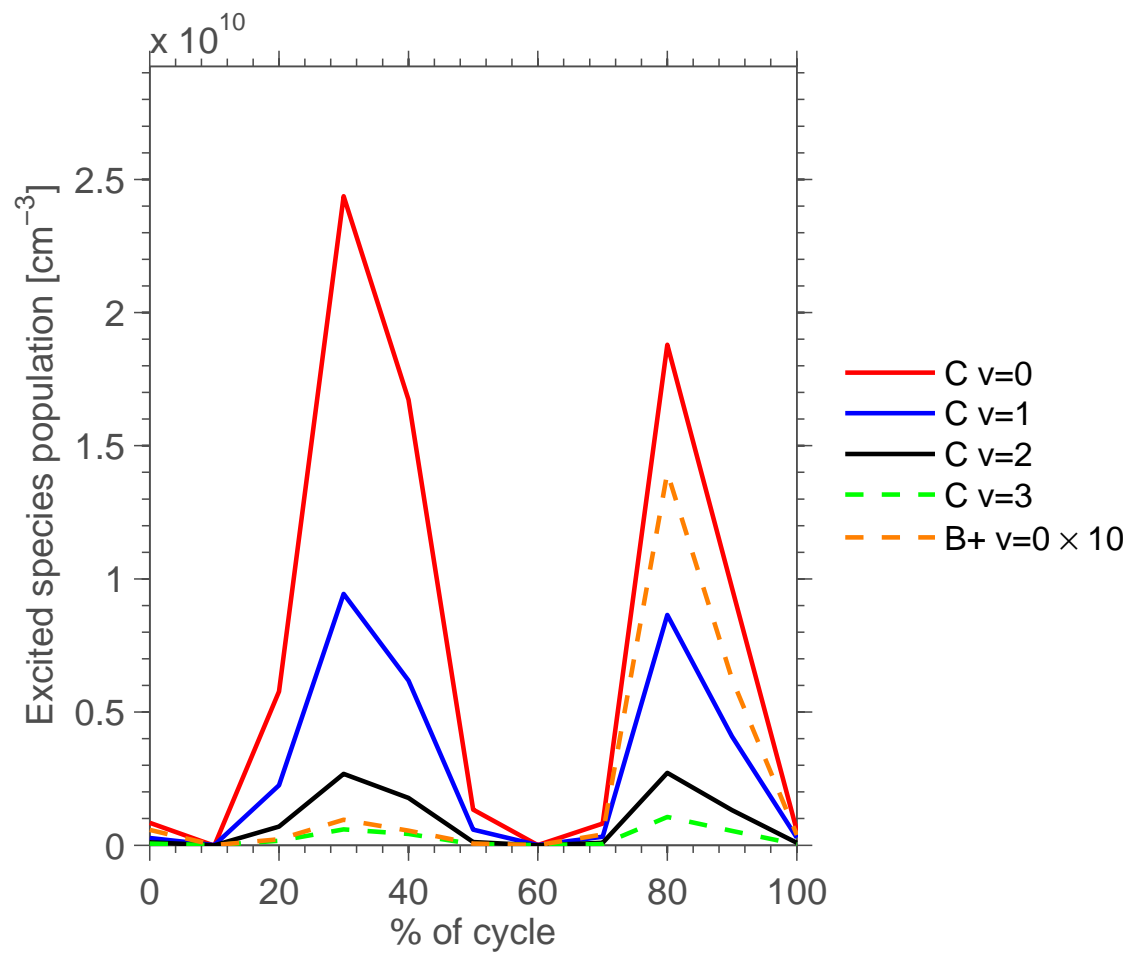

Figure 11. Excited species population over cycle time for $15 \mathrm{kV}$ and $2.5 \mathrm{kHz}$ at atmospheric pressure. $\mathrm{C} \mathrm{v}=0,1,2,3$ and $\mathrm{B}+\mathrm{v}=0$ represent excited species population density of $\mathrm{N}_{2}\left(C^{3} \Pi_{\mathrm{u}}, v=0,1,2,3\right)$ and $\mathrm{N}_{2}^{+}\left(B^{2} \Sigma_{\mathrm{u}}^{+}, \mathrm{v}=0\right)$ states respectively.

In Eq. (1), $K_{i}(E / N)$ is the production rate for the electron-impact excitation process $i$, which depends on $\mathrm{E} / \mathrm{N} . X_{i}$ is the target gas density. $\tau_{i}$ is the total lifetime of the excited state $i$, calculated as $\tau_{i}=$ $1 / \tau_{0}^{i}+Q_{\mathrm{N}_{2}}^{i} N_{2}+Q_{\mathrm{O}_{2}}^{i} \mathrm{O}_{2}$, where $\tau_{0}^{i}$ is the radiative lifetime of the species $i$ and $Q_{\mathrm{X}}^{i}$ is the quenching rate of the $i^{\text {th }}$ state by the quencher X. From Eq. (1), Eq. (2) is obtained.

$$
0=n_{\mathrm{e}} X_{i} K_{i}(E / N)-\frac{n_{i}}{\tau_{i}}
$$

The experimental determination of the reduced electric field can be performed by comparing the relative population of two different excited species. By dividing Eq. (2) for two different excited species, an equation in which only the reduced electric field is unknown is obtained. The production rates for the excitation process are computed using Eq. (3).

$$
K_{i}(E / N)=\frac{2 \mathrm{e}}{m} \int_{0}^{\infty} \varepsilon \sigma_{i}^{\mathrm{e}} F_{0} d \varepsilon
$$

In Eq. (3), $\varepsilon=m v^{2} /(2 e)$ is the kinetic energy of the electron, $F_{0}$ is the electron energy distribution function (EEDF), which is computed in the Boltzmann solver Bolsig $+{ }^{13}$ and $\sigma_{i}^{e}$ is the excitation cross section. The references corresponding to the excitation cross sections and quenching rates of the excited species used in this study are shown in table 3 . The resulting plots for reduced electric field strength for atmospheric and low pressure are shown in figures 12 and 13 respectively.

In figure 12, the reduced electric field is plotted for different voltages and frequencies. The value of the reduced electric field differs during rise and fall time of the voltage signal. During the rise time, the reduced electric field is approximately $800 \mathrm{Td}$, whereas during fall time, it only reaches about $200 \mathrm{Td}$. This behaviour is observed for all measured conditions. Furthermore, at the highest voltage $(15 \mathrm{kV})$, the breakdown electric field value is reached earlier in the cycle. This corresponds to the current signal measurements in table 1 and image intensity in figure 8 . However, this behaviour is only observed during fall time. A different trend 


\begin{tabular}{lll} 
Process & Reference for e-impact & Reference for quenching \\
\hline $\mathrm{N}_{2}\left(X^{1} \Sigma_{g}^{+}\right)+\mathrm{e} \rightarrow \mathrm{N}_{2}\left(\mathrm{~B}^{3} \Pi_{\mathrm{g}}\right)+\mathrm{e}$ & 14 & 15 \\
$\mathrm{~N}_{2}\left(X^{1} \Sigma_{g}^{+}\right)+\mathrm{e} \rightarrow \mathrm{N}_{2}\left(\mathrm{C}^{3} \Pi_{\mathrm{u}}\right)+\mathrm{e}$ & 14 & 16,17 \\
$\mathrm{~N}_{2}\left(X^{1} \Sigma_{g}^{+}\right)+\mathrm{e} \rightarrow \mathrm{N}_{2}^{+}\left(\mathrm{B}^{2} \Sigma_{\mathrm{u}}^{+}, \mathrm{v}=0\right)+2 \mathrm{e}$ & 14 & 18 \\
Ar $($ ground $)+\mathrm{e} \rightarrow \operatorname{Ar}\left(2 \mathrm{p}_{1}\right)+\mathrm{e}$ & 19 & 20 \\
\hline
\end{tabular}

Table 3. Electron-impact excitations and references for the corresponding excitation cross sections and quenching rates.

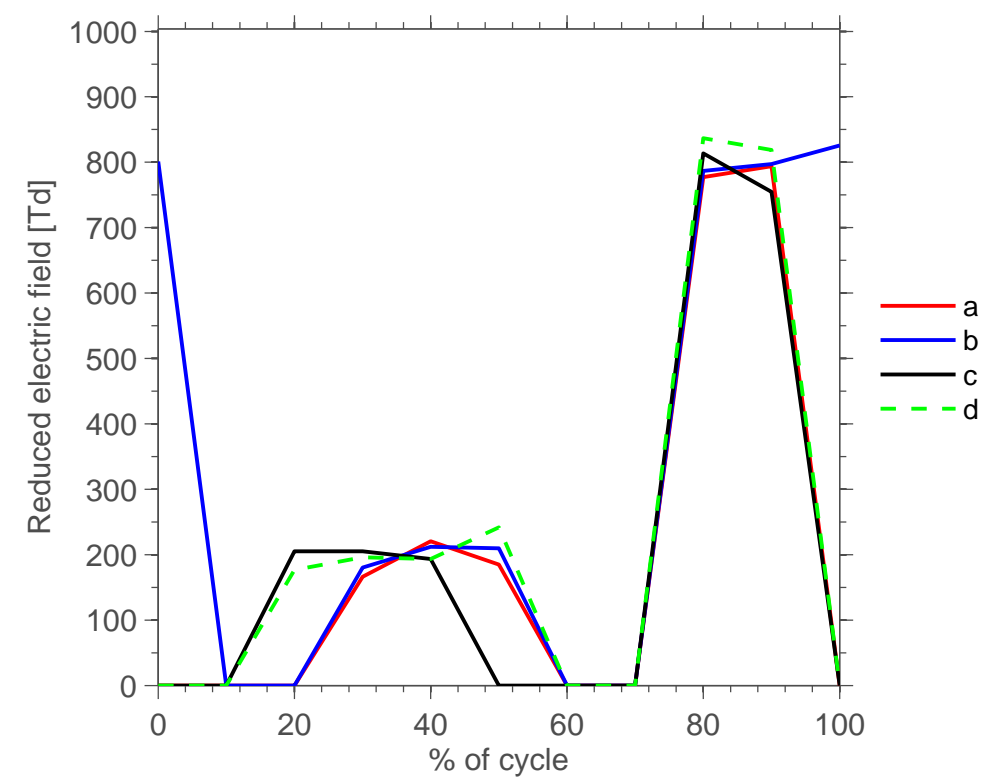

Figure 12. Reduced electric field over cycle time for a) $10 \mathrm{kV}, 2.5 \mathrm{kHz} \mathrm{b}) 10 \mathrm{kV}, 3.5 \mathrm{kHz} \mathrm{c}) 15 \mathrm{kV}, 2.5 \mathrm{kHz} \mathrm{d}) 15 \mathrm{kV}$, $3.5 \mathrm{kHz}$. Measurements performed at atmospheric pressure.

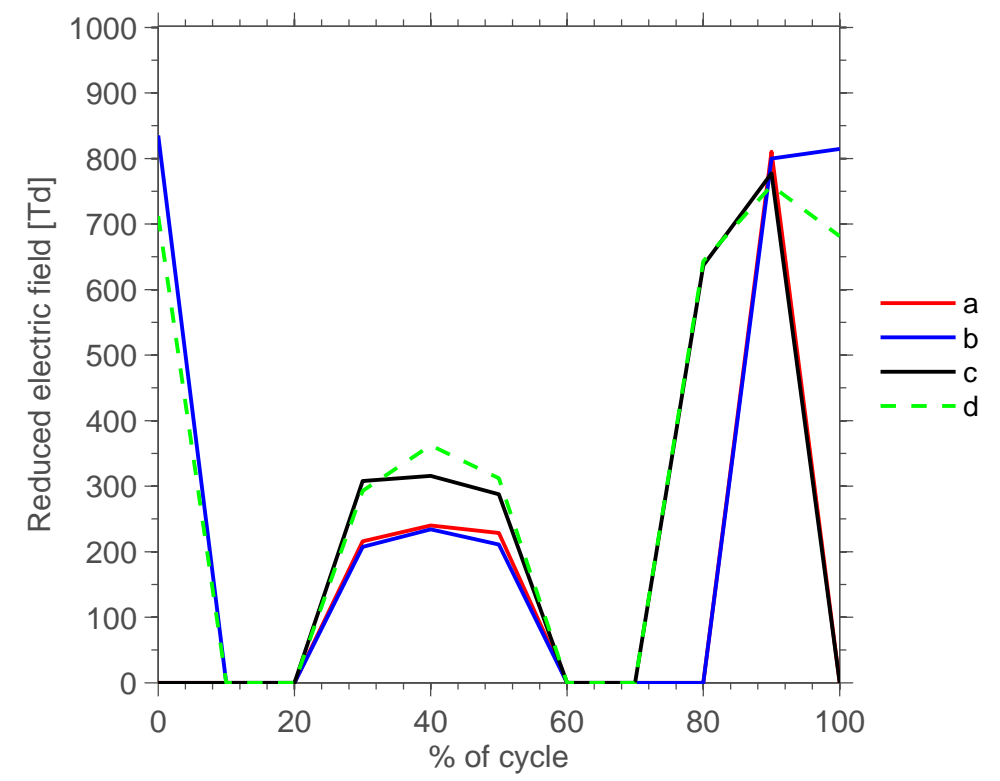

Figure 13. Reduced electric field over cycle time for a) $7.5 \mathrm{kV}, 2.5 \mathrm{kHz}, 500$ mbar b) $7.5 \mathrm{kV}, 3.5 \mathrm{kHz}, 500 \mathrm{mbar}$ c) $5 \mathrm{kV}$, $2.5 \mathrm{kHz}, 250$ mbar d) $5 \mathrm{kV}, 3.5 \mathrm{kHz}, 250$ mbar.

is observable in figure 13, where the electric field value starts increasing earlier in the cycle during rise time for the cases with highest voltage over pressure ratio. 


\section{Conclusions}

Plasma characteristics of the NATO-AVT-RTO-190 AC-DBD test case were analysed experimentally. Current signal measurements revealed information about the strength, duration and starting time of the plasma for different voltages and frequencies. A higher voltage or frequency shifts the starting point of the plasma towards the beginning of the cycle, as the breakdown voltage is reached earlier. The number of current peaks increases with rising voltage or frequency.

Thrust and power consumption measurements were performed for different driven voltages and frequencies. The results showed that the force generated by the actuator increases as the driven frequency or voltage increases. An optimal condition for operating the SDBD plasma actuator exists, and pushing for higher voltage levels is not necessary.

Detailed fast-camera imaging and spectroscopy allows understanding of the plasmas transitions and characteristics, as well as their temporal evolution with respect to different operating conditions of voltage, frequency and ambient pressure. From optical emission spectroscopy, the excited species in the plasma are detected and the reduced electric field is calculated. These are in agreement with trends noticed during current measurements and plasma imaging.

This study allows to characterise SDBD plasma and contributes to understanding the plasma physics which are believed to be related to aerodynamic control.

\section{Acknowledgments}

The research was performed within the Basic Plasma Physics and Plasma Processing Group at the Centre for Plasma Physics at EPFL. The authors would like to thank Christoph Hollenstein, Alan Howling and Ivo Furno for their support and providing the available equipment. The research at DRDC Valcartier and Laval University was funded by a DND TIF project.

\section{References}

\footnotetext{
${ }^{1}$ Corke, T., Enloe, C., and Wilkinson, S., "Dielectric Barrier Discharge Plasma Actuators for Flow Control," Annual Review of Fluid Mechanics, Vol. 42, No. 1, Jan. 2010, pp. 505-529.

${ }^{2}$ Moreau, E., Louste, C., and Touchard, G., "Electric wind induced by sliding discharge in air at atmospheric pressure," Journal of Electrostatics, Vol. 66, No. 1-2, Jan. 2008, pp. 107-114.

${ }^{3}$ Pavon, S., Dorier, J. L., Hollenstein, C., Ott, P., and Leyland, P., "Effects of high-speed airflows on a surface dielectric discharge," Journal of Physics D: Applied Physics, Vol. 40, No. 6, 2007.

${ }^{4}$ Pavon, S., Ott, P., Leyland, P., Dorier, J. L., and Hollenstein, C., "Effects of a surface dielectric barrier discharge on transonic flows around an airfoil," AIAA 2009-649, 47th AIAA Aerospace Sciences Meeting, Orlando, 2009.

${ }^{5}$ Roupassov, D. V., Nikipelov, A. A., Nudnova, M. M., and Starikovskii, A. Y., "Flow Separation Control by Plasma Actuator with Nanosecond Pulsed-Periodic Discharge," AIAA Journal, Vol. 47, No. 1, 2009.

${ }^{6}$ Starikovskii, A. Y., Nikipelov, A. A., Nudnova, M. M., and Roupassov, D. V., "SDBD plasma actuator with nanosecond pulse-periodic discharge," Plasma Sources Sci. Technol., Vol. 18, No. 3, 2009.

${ }^{7}$ Little, J., Takashima, K., Nishihara, M., Adamovich, I., and Samimy, M., "High lift airfoil leading edge separation control with nanosecond pulse driven DBD plasma actuators," AIAA 2010-4256, 5th AIAA Flow Control Conference, Chicago, 2010, Chicago, 2010.

${ }^{8}$ Grech, N., Peschke, P., and Leyland, P., "Investigation of Flow Separation Control by Nanosecond Pulsed Dielectric Barrier Discharge Actuators," Progress in Flight Physics Volume 6: Selected papers of the EUCCAS 2013 5th European Conference for Aeronautics and Space Sciences, Munich, Germany, 2013., 2014.

${ }^{9}$ Pavón, S., "Interaction between a surface dielectric barrier discharge and transonic airflows," EPFL thesis, Vol. $4201,2008$. ${ }^{10}$ Whiting, E. E. and Center, A. R., Computer program for determining rotational line intensity factors for diatomic molecules, National Aeronautics and Space Administration, 1973.

${ }^{11}$ Gilmore, F. R., Laher, R. R., and Espy, P. J., "Franck-Condon Factors, r-Centroids, Electronic Transition Moments, and Einstein Coefficients for Many Nitrogen and Oxygen Band Systems," Journal of Physical and Chemical Reference Data, Vol. 21, No. 5, 1992, pp. 1005-1107.

${ }^{12}$ Hornkohl, J. O., Parigger, C. G., and Nemes, L., "Diatomic Hönl-London factor computer program," Appl Opt, Vol. 44, No. 18, June 2005, pp. 3686-3695, PMID: 15989043.

${ }^{13}$ Hagelaar, G. J. M. and Pitchford, L. C., "Solving the Boltzmann equation to obtain electron transport coefficients and rate coefficients for fluid models," Plasma Sources Science and Technology, Vol. 14, No. 4, Nov. 2005, pp. 722-733.
} 
${ }^{14}$ Tabata, T., Shirai, T., Sataka, M., and Kubo, H., "Analytic cross sections for electron impact collisions with nitrogen molecules," Atomic Data and Nuclear Data Tables, Vol. 92, No. 3, May 2006, pp. 375-406.

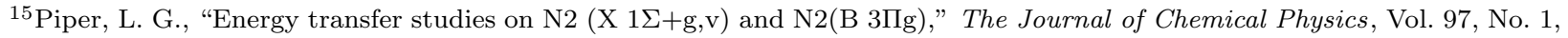
July 1992, pp. 270-275.

${ }^{16}$ Dilecce, G., Ambrico, P. F., and Benedictis, S. D., "New N $2\left(C^{3} \Pi \mathrm{u}, v\right)$ collision quenching and vibrational relaxation rate constants: 2. PG emission diagnostics of high-pressure discharges," Plasma Sources Science and Technology, Vol. 16, No. 1, Feb. 2007, pp. S45-S51.

${ }^{17}$ Pancheshnyi, S. V., Starikovskaia, S. M., and Starikovskii, A. Y., "Measurements of rate constants of the N2(C3חu, v'=0) and $\mathrm{N} 2+(\mathrm{B} 2 \Sigma+\mathrm{u}, \mathrm{v}$ ' $=0)$ deactivation by $\mathrm{N} 2, \mathrm{O} 2, \mathrm{H} 2, \mathrm{CO}$ and $\mathrm{H} 2 \mathrm{O}$ molecules in afterglow of the nanosecond discharge," Chemical Physics Letters, Vol. 294, No. 6, Sept. 1998, pp. 523-527.

${ }^{18}$ Valk, F., Aints, M., Paris, P., Plank, T., Maksimov, J., and Tamm, A., "Measurement of collisional quenching rate of nitrogen states N2(C 3חu, v =0) and N2+ B2," J. Phys. D: Appl. Phys., Vol. 43, No. 38, Sept. 2010, pp. 385202.

${ }^{19}$ Boffard, J. B., Chiaro, B., Weber, T., and Lin, C. C., "Electron-impact excitation of argon: Optical emission cross sections in the range of 300-2500nm," Atomic Data and Nuclear Data Tables, Vol. 93, No. 6, Nov. 2007, pp. 831-863.

${ }^{20}$ Sadeghi, N., Setser, D. W., Francis, A., Czarnetzki, U., and Döbele, H. F., "Quenching rate constants for reactions of $\operatorname{Ar}(4 \mathrm{p}$ '[1/2]0, 4p[1/2]0,4p[3/2]2, and 4p[5/2]2) atoms with 22 reagent gases," The Journal of Chemical Physics, Vol. 115, No. 7, Aug. 2001, pp. 3144-3154. 